\title{
Approaches to Classification of Multichannel Images
}

\author{
Vladimir Lukin ${ }^{1}$, Nikolay Ponomarenko ${ }^{1}$, Andrey Kurekin ${ }^{2}$, Kenneth Lever ${ }^{2}$, \\ Oleksiy Pogrebnyak ${ }^{3}$, and Luis Pastor Sanchez Fernandez ${ }^{3}$ \\ ${ }^{1}$ National Aerospace University, Dept of Transmitters, Receivers and Signal Processing, \\ 17 Chkalova St, 61070 Kharkov, Ukraine \\ lukin@xai.kharkov.ua, uagames@mail.ru \\ ${ }^{2}$ Department of Computer Science, School of Engeneering, Cardiff University, \\ 5 The Parade, Roath, Cardiff CF24 3AA, UK \\ A.Kurekin@ Cs. cardiff.ac.uk \\ ${ }^{3}$ Instituto Politecnico Nacional, Centro de Investigacion en Computacion, Ave. Juan de Dios \\ Batiz S/N, C.P. 07738, Mexico, D.F., Mexico \\ olek@pollux.cic.ipn.mx
}

\begin{abstract}
The comparison of different approaches to classification of multichannel remote sensing images obtained by spaceborne imaging systems is presented. It is demonstrated that it is reasonable to compress original noisy images with appropriate compression ratio and then to classify the decompressed images rather than original data. Two classifiers are considered: based on radial basis function neural network and support vector machine. The latter one produces slightly better classification results.
\end{abstract}

Keywords: Multichannel image classification, image compression, remote sensing.

\section{Introduction}

Nowadays airborne and spaceborne remote sensing (RS) systems produce a lot of data that are valuable for such applications like ecological monitoring, forestry, agriculture, catastrophe prediction, etc [1]. Most of such systems operate in the so called multichannel mode [2]. Term "multichannel" means that for some terrain lot several component images are formed in different bands or using different polarizations of transmitted and/or received signals. Multichannel RS systems are potentially able to provide more reliable retrieval of useful information from RS data and/or more accurate estimation of sensed terrain parameters.

Multichannel data can be transferred from airborne or spaceborne carrier to an onground processing center without any compression, coded in a lossless manner, or lossy compressed. In case of lossless coding the provided compression ratio (CR) is commonly within the limits from 2 to $3[3,4]$ that often is inappropriate. Recently, several papers that report on possibilities of applying lossy compression to multi- and hyperspectral images have appeared (see, e.g., [5-7]). It has been demonstrated that quite good classification of multichannel RS data can be provided even if the used CR is from 20 to 50 . It can be explained by the fact that the original multichannel $\mathrm{RS}$ 
images often are noisy [8] and noise suppression using image compression techniques leads to increasing the reliability of correct classification simultaneously decreasing the data size [8-10]. The only important item is that CR should be adjusted in correspondence with noise variance for providing a proper trade-off between noise suppression and distortions introduced due to lossy compression $[9,10]$. This allows expecting that the probability of correct classification of multichannel RS data can be of the same order for two cases: a) applying classification procedure to original (noisy) data; b) applying classification to data compressed in a lossy manner with proper selection of a compression ratio.

The aim of this paper is twofold. The first goal is to compare both aforementioned approaches and to make up the corresponding practical recommendations. The second goal is to consider two modern classifier types based on using support vector machines (SVM) and radial basis function (RBF) neural networks $(\mathrm{NN})$, both trained in a supervised manner.

\section{Lossy Compression of Noisy Images}

First, it is worth noting that in case of lossy compression of noisy images it is reasonable to use not typical criteria of compressed image quality $[9,10]$. Recall that if one deals with traditional lossy compression of still images, the compression is basically characterized by the rate-distortion curve (PSNR vs CR) [11]. PSNR or for decompressed image is calculated with respect to the corresponding original image. On the contrary, if a noisy image is subject to compression, it is also expedient to control similarity of decompressed and noise-free images that can be characterized by PSNR $_{\text {nf }}$ calculated with the use of MSE between decompressed and noise free images. Certainly, PSNR $\mathrm{nf}_{\mathrm{n}}$ can be obtained by simulations with test images to which noise has been artificially added.

Fig. 1 presents the corresponding curves for the test gray-scale image Lena corrupted by additive, zero mean Gaussian noise with variance $\sigma^{2}=50$. Two compression methods have been considered, the standard JPEG 2000 [12] and the recently introduced AGU coder [13].

For both considered coders the dependencies of $\mathrm{PSNR}_{\text {or }}$ behave in conventional manner, i.e., PSNR $_{\text {or }}$ becomes smaller when the compression rate increases. The behavior of the PSNR $\mathrm{Pf}_{\mathrm{n}}$ curve is more specific. As seen, for both considered coders $\mathrm{PSNR}_{\mathrm{nf}}$ increases when $\mathrm{CR}$ reduces from 2 to approximately $0.35 \mathrm{bpp}$. At $\mathrm{CR} \approx 0.35 \mathrm{bpp}$ the maximums for both curves are observed, and further reduction of $\mathrm{CR}$ leads to rapid $\mathrm{PSNR}_{\mathrm{nf}}$ decreasing. Such a compression rate for which the maximum of $\mathrm{PSNR}_{\mathrm{nf}}$ is observed called optimal operation point (OOP). Intuitively, this can be favorable for image classification.

As shown in [9], for OOP determination one should know in advance or to preestimate the noise characteristics, and it can be done in a blind manner [15, 16]. Approaches to provide OOP in an automatic manner have been proposed in $[9,17]$.

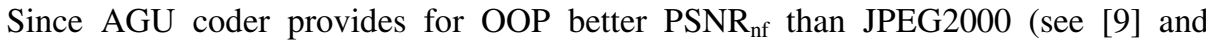
particular data in Fig. 1), below we will consider the application of AGU coder for compressing multichannel images. 


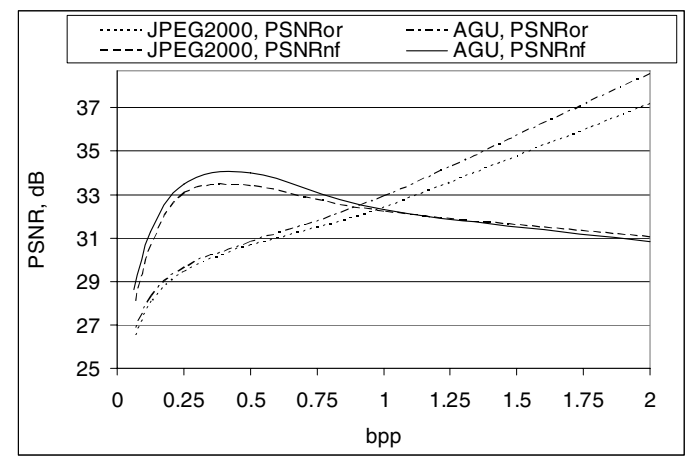

Fig. 1. Dependencies PSNR ${ }_{\text {nf }}$ and $\mathrm{PSNR}_{\text {or }}$ vs CR for JPEG2000 and AGU techniques for test image Lena corrupted by additive noise with $\sigma^{2}=50$

\section{Considered Approaches to Classification of Original Noisy and Compressed Images}

Classification has been applied to the three-channel test image presented in Fig. 2,a. This image has been obtained by using Landsat TM images of three bands (ftp://ftp.glcf.umiacs.umd.edu/glcf/Mosaic_Landsat), for which the corresponding ground truth map is available.

There are five basic classes in the observed scene, namely, soil, grass, water surface, roads and urban areas, bushes. The percentage of pixels (more exactly, voxels) belonging to each class is rather different and it varies from $7.4 \%$ for roads and urban areas to $43.6 \%$ for grass. Two classifiers have been employed. The first one is based on Support Vector Machines (SVM) that has superior performance comparing to many standard classification techniques in various applications [18].

The SVM classifier uses a "kernel trick" method for mapping the original feature space of classified data into a higher-dimensional non-linear feature space. For

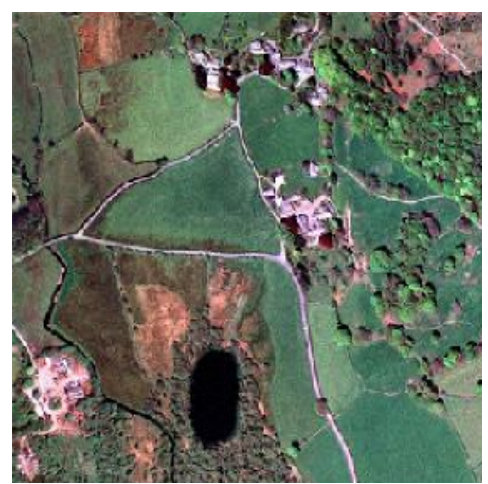

a

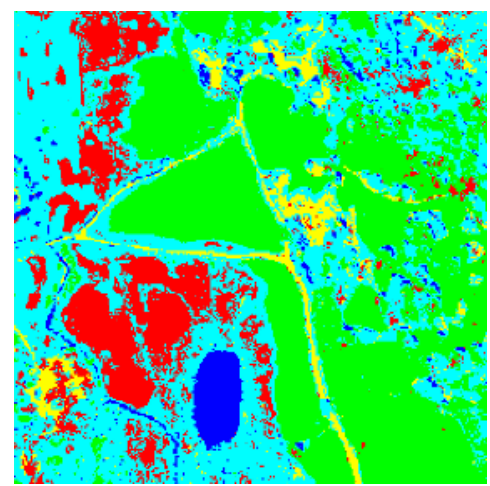

b

Fig. 2. The test three-channel image in RGB representation (a) and the corresponding classification map for noise-free multichannel data (b) 
two-class (binary) classification problem the SVM classifier uses a hyperplane to subdivide the feature space into two regions and attributes the input feature vector to one of two classes. There are different ways for choosing a hyperplane in a feature space. One of them is a maximum margin approach that maximizes the distance from the hyperplane to the nearest training samples in a feature vector space. This approach is not applicable for training data with classification errors and data corrupted by noise, such as multichannel RS images considered in this paper. Obviously, classification errors are natural for some part of noisy data used for SVM training, and maximum margin condition can not be satisfied for all vectors of the training data set.

For training the SVM classifier we used a more practical approach. It is based on choosing a soft margin hyperplane that allows a small amount of errors in training data by introducing slack variables in the objective function. For binary classification the soft margin hyperplane is found by solving the primary optimization problem [18]

$$
\min _{\mathbf{w}, b, \xi_{n}}\left\{\frac{1}{2} \mathbf{w}^{T} \mathbf{w}+\sum_{n=1}^{N} C \xi_{n}\right\}
$$

subject to

$$
\begin{aligned}
y_{n}\left(\mathbf{w}^{T} \varphi\left(\mathbf{x}_{n}\right)+b\right) & \geq 1-\xi_{n}, \\
\xi_{n} & \geq 0 \quad \text { for } i=1, \ldots, N
\end{aligned}
$$

where $N$ is a number of training samples, $\left\{\left(\mathbf{x}_{n}, y_{n}\right)\right\}_{n=1}^{N}$ is the training dataset and $y_{n}=1$ for the first class and $y_{n}=-1$ for the second class of training data. $\xi_{n}$ are slack variables. If $\xi_{n}>0$, the slack variables indicate training data with classification errors. $C>0$ is an error penalty parameter that determines the trade-off between the hyperplane margin and classification errors; $\mathbf{w}$ and $b$ are the parameters of hyperplane $\left(\mathbf{w} \cdot \mathbf{x}_{n}\right)+b=0$. By introducing Lagrange multipliers $\alpha_{n} \geq 0$, the constrained optimization problem (1) can be expressed as dual optimization problem [20]

$$
\max _{\alpha_{n}}\left\{\sum_{n=1}^{N} \alpha_{n}-\frac{1}{2} \sum_{m, n=1}^{N} \alpha_{m} \alpha_{n} y_{m} y_{n} \varphi\left(\mathbf{x}_{m}\right)^{T} \varphi\left(\mathbf{x}_{n}\right)\right\}
$$

subject to the constraints

$$
\sum_{n=1}^{N} \alpha_{n} y_{n}=0, \text { and } C \geq \alpha_{i} \geq 0 \text { for } n=1, \ldots, N
$$

where $\alpha_{n} \geq 0$. For Lagrange multipliers $\alpha_{n}$ not equal to zero the corresponding training vectors are named "support vectors".

The dot product of two feature vectors in (3) is calculated indirectly by application of "kernel trick" method. A kernel function $K(\mathbf{x}, \mathbf{y})$ is expressed as

$$
K(\mathbf{x}, \mathbf{y})=\varphi(\mathbf{x})^{T} \varphi(\mathbf{y})
$$

where function $K(\mathbf{x}, \mathbf{y})$ is subject to Mercer's condition [18, 20]. One of the frequently used kernel functions is a radial basis function (RBF)

$$
K(\mathbf{x}, \mathbf{y})=\exp \left(-\gamma\|\mathbf{x}-\mathbf{y}\|^{2}\right), \gamma>0
$$


where $\gamma$ is the function control parameter. The RBF kernel has only one parameter, it provides the same performance as linear kernel, for certain parameters it behaves like some other kernel types and is a reasonable choice for the considered classification task. Application of (5) for a hyperplane equation gives the output of SVM classifier

$$
f(\mathbf{x})=\operatorname{sgn}\left(\sum_{n=1}^{N} y_{n} \alpha_{n} K\left(\mathbf{x}, \mathbf{x}_{n}\right)+b\right)
$$

where $\mathbf{x}$ is the input feature vector. For the considered scenario of classifying threechannel test images, the feature vector is composed of three pixel values for RGB image components $\mathbf{x}=\left(x^{R}, x^{G}, x^{B}\right) \in R^{3}$.

The SVM classifier given by equations (1)-(7) is designed for solving binary classification tasks. Several approaches have been proposed for solving multi-class problems based on direct classification of all data in one optimization formulation and combining several binary SVM classifiers [21]. The first one has to deal with all SVM parameters for the objective function optimization and it is usually more computationally expensive than the second approach that we applied for classification of the test image in Fig.2,a. It is based on one-against-one classification strategy that divides multi-class problem into $K(K-1) / 2$ separate binary classification problems for all pair combinations of $K$ classes. The binary classification problems are solved by SVM classifiers and a majority voting rule is applied to find the output class [21].

While the number of class training samples is not equal, we used different error penalty parameters $C_{i}$ in the objective function (1) to provide equal contributions from every image class and achieve similar probability of correct classification. For $i$-th class the parameter $C_{i}$ is given by

$$
C_{i}=C^{*} \frac{N}{K N_{i}}
$$

where $C^{*}$ is a constant. $N_{i}$ is a number of training samples for class $i$ and $N$ is a total number of samples. The error parameter $C^{*}$ and the RBF control parameters are evaluated by cross-validation [19].

Another approach presumes the use of radial basis function (RBF) neural network (NN) classifier [22]. The RBF NN demonstrates high efficiency, low computational demands and simplicity of software and hardware implementation at training and classification stages [23, 24]. The RBF NN classifier we used had an input layer, single hidden layer with nonlinear neurons and an output layer with linear neurons. The number of neurons in the input layer was the same as the number of input features, and the number of output layer neurons was equal to the number of data classes. The number of neurons in the hidden layer depends on such factors, as classification complexity and feature space dimensionality. The activation function of the hidden layer neurons had the same form as the RBF kernel function (6) we considered for SVM classifier. The training of RBF NN was performed by minimizing the mean square error $E$ of the $\mathrm{NN}$ output

$$
E=\frac{1}{K N} \sum_{k=1}^{K} w_{k} \sum_{n=1}^{N}\left(Z_{n, k}-y_{n, k}\right)^{2}
$$


where $Z_{n, k}$ is the output of the $\mathrm{NN}$ output layer representing the accumulated membership of the $n$-th data sample to the object class $k . y_{n, k}$ is the desired output of neural network, $N$ is the number of training samples and $K$ is the number of classes. The error function (9) was weighted by $w_{k}$ values to provide equal contributions from every image class for the different numbers of class training samples. The weight for every class is equal to the reciprocal of the number of class training samples.

A cascade-correlation algorithm that provides nearly optimal training results with the extended number of neurons in the RBF NN hidden layer was used to minimize the objective function (9). Training starts with one hidden layer neuron and the new ones are added iteratively to reduce the total residual error. The weighting coefficients of new hidden neuron are selected to provide the condition of maximum correlation between the new unit output and the trained NN output error [25]. The optimal number of hidden layer neurons is selected automatically by cross validation [19].

Classifiers have been trained in a supervised manner using small portions of images from 181 to 1610 pixels depending upon the class. In Fig. 3 the lots from which the training sets were taken are shown. After learning, the classifiers have been applied to the entire image. The pixels to which classification has been applied are depicted by black color in Fig. 3 .

Learning of classifiers has been performed for each particular case of the test image. First, it was done for the original noisy image and, second, for the image compressed with optimal CR. Note that different sets of classifier parameters have been obtained for these cases. Two values of additive Gaussian noise variance have been used in simulations: 49 and 100 for byte component images. In the first case the optimal CR was about 8 , and for $\sigma^{2}=100$ the optimal $C R \approx 10.7$.

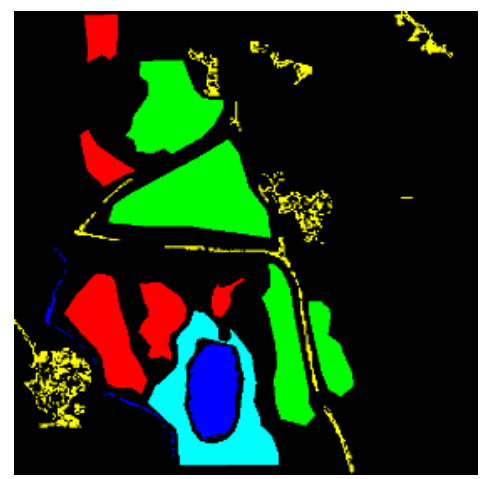

Fig. 3. The lots (image fragments) used for training the SVM and RBF NN classifiers

\section{Experimental Result Analysis}

The results obtained for SVM classifier for both variance values $\left(\sigma^{2}=49\right.$ and $\left.\sigma^{2}=100\right)$ are presented in Table 1. For the class bare soil we have obtained $87.25 \%$ of correctly classified pixels, for grass $99.71 \%$, for water surface $-98.38 \%$, for roads and urban areas $-67.34 \%$, and for bushes $-87.13 \%$ with overall accuracy $\mathrm{P}_{\text {cor ov }}=91.48 \%$. As 
seen, the lowest probability of correct classification is observed for classes for which RS data are either rather heterogeneous of appear themselves as prolonged or small size objects.

Some tendencies observed from data in Table 1 are trivial and expectable. In particular, the probabilities $\mathrm{P}_{\text {cor } \mathrm{k}}$ for particular classes as well as $\mathrm{P}_{\text {cor ov }}$ for noisy images are smaller than for noise-free data irrespectively is classification applied to original images or to compressed ones. Practically for all classes increasing of $\sigma^{2}$ leads to decreasing of $\mathrm{P}_{\text {cor } \mathrm{k}}$ and, respectively, these results in reduction of $\mathrm{P}_{\text {cor ov }}$.

Table 1. Precentage of correctly classified pixels for the trained SVM

\begin{tabular}{|c|c|c|c|c|c|c|c|}
\hline $\begin{array}{c}\text { Noise } \\
\text { variance }\end{array}$ & $\begin{array}{c}\text { Classified } \\
\text { image }\end{array}$ & $\begin{array}{c}\text { Bare } \\
\text { soil }\end{array}$ & Grass & Water & Roads & Bushes & Overall \\
\hline 49 & Original & 82.00 & 94.37 & 96.17 & 67.62 & 71.27 & 85.46 \\
\hline 49 & Compressed & 83.39 & 91.57 & 95.59 & 65.60 & 81.43 & 85.80 \\
\hline 100 & Original & 74.90 & 91.68 & 94.24 & 59.81 & 67.30 & 81.13 \\
\hline 100 & Compressed & 83.43 & 93.43 & 92.76 & 62.07 & 76.23 & 85.21 \\
\hline
\end{tabular}

The most interesting fact is that for both considered values of $\sigma^{2}$ the probabilities $P_{\text {cor ov }}$ are larger if classification is applied to compressed RS data. For the case $\sigma^{2}=100$ the benefit attained due to classifying compressed data in comparison to classifying original multichannel images is considerable. This means that a positive effect due to the noise suppression taking place in case of image compression is larger than a negative effect due to the introduced distortions. Concerning particular classes, sometimes better values of $\mathrm{P}_{\text {cor } \mathrm{k}}$ are provided if classification is applied to the original data.

The classification maps for applying the SVM based approach to original and compressed data in the case of $\sigma^{2}=100$ are presented in Fig 4,a, and Fig. 4,b, respectively. Comparison of these classification maps between each other and to the more accurate classification map (Fig. 2,b) shows the following. Being applied pixelwise to original noisy image, the classification results in quite many misclassifications represented as separate pixels surrounded by the pixels referred to another class (see Fig. 4,a). This effect appears in less degree if trained SVM classifier is applied to the compressed data.

The classification results for RBF NN are presented in Table 2. For all cases of $\mathrm{NN}$ training the number of hidden units was 17 . The percentages of correctly classified pixels are equal to $82.42,99.67,97.76,71.72,85.14 \%$ for the classes bare soil, grass, water, roads and urban areas, and bushes, respectively; $\mathrm{P}_{\text {cor ov }}=0.9062$. As seen, these probabilities are, in general, slightly worse than for SVM classifier.

The obtained results are quite similar to those ones presented in Table 1. The values $\mathrm{P}_{\text {cor ov }}$ for the corresponding classifiers and noise variances differ by less than 0.01. SVM recognizes better the class "soil" while RBF NN provides better classification of water surface. Again, the overall percentage of correctly classified pixels is larger if the classifier is applied to the compressed RS data. And the difference becomes more evident if the noise variance increases (see data for $\sigma^{2}=100$ ). 


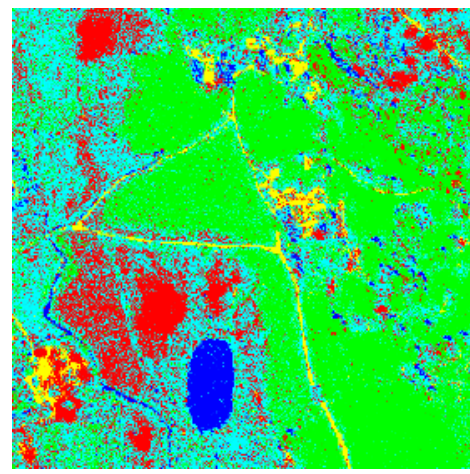

a

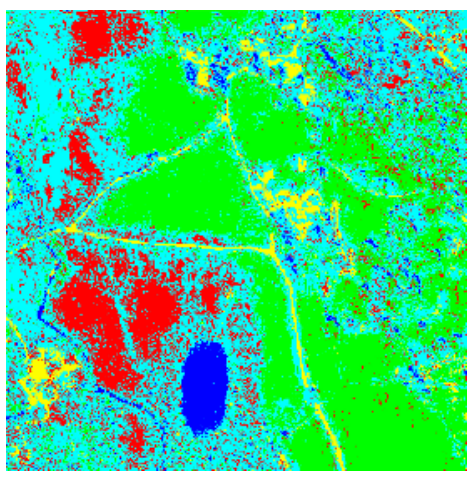

b

Fig. 4. Classification maps for SVM applied to original (a) and compressed (b) RS data

Table 2. Precentage of correctly classified pixels for the trained RBF NN

\begin{tabular}{|c|c|c|c|c|c|c|c|}
\hline $\begin{array}{c}\text { Noise } \\
\text { variance }\end{array}$ & $\begin{array}{c}\text { Classified } \\
\text { image }\end{array}$ & $\begin{array}{c}\text { Bare } \\
\text { soil }\end{array}$ & Grass & Water & Roads & Bushes & $\begin{array}{c}\text { Overa } \\
\text { ll }\end{array}$ \\
\hline 49 & Original & 75.22 & 95.81 & 99.48 & 66.50 & 75.51 & 85.41 \\
\hline 49 & Compressed & 76.41 & 94.95 & 99.56 & 65.41 & 80.80 & 85.98 \\
\hline 100 & Original & 73.62 & 91.58 & 99.66 & 67.14 & 65.50 & 81.79 \\
\hline 100 & Compressed & 74.59 & 93.21 & 99.52 & 64.24 & 78.29 & 84.32 \\
\hline
\end{tabular}

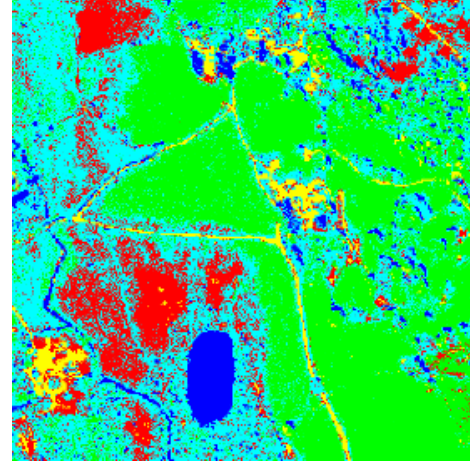

a

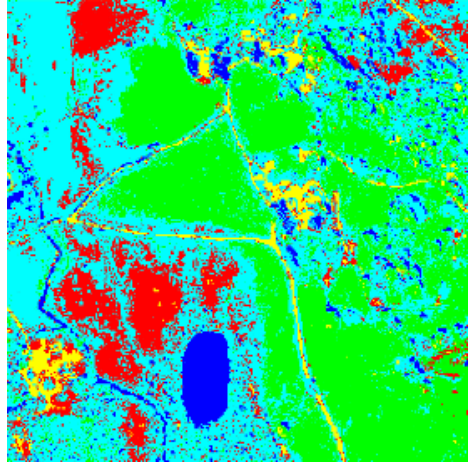

b

Fig. 5. Classification maps for RBF NN applying to original (a) and compressed (b) RS data

Examples of multichannel image classification using RBF NN for $\sigma^{2}=49$ are given in Fig. 5. There are some noticeable differences in the spatial structure of the classified data. In particular, there are more separate pixel misclassifications if RBF NN classifier is applied to the original noisy data (see Fig. 5,a). The most problematic class is "roads and urban areas". Both classifiers recognize the corresponding regions with the smallest probability of correct classification. One possible reason is that we have considered pixel-by-pixel classification without taking into account any spatial features. Besides, no post-processing of the obtained primary classification maps has 
been utilized. These opportunities for further classification improving can be subject of the future work.

\section{Conclusions}

Two approaches to classification of multichannel RS data are considered. It is shown that in case of presence of rather intensive noise in obtained images it is reasonable to perform on-board lossy compression and then to apply on-land classification of decompressed images instead of data lossless compression and using the classification of original noisy images. The CR provided for such lossy compression can be about 10 and this is considerably better than in case of lossless coding. Moreover, we considered component-wise compression of multichannel images. And if the noise level is approximately the same in all component images, the same quantization step can be used in compressing multichannel images as multidimensional data. This can lead to further improvement of CR for given performance of the coder.

The obtained results and observed tendencies are in good coincidence for two different approaches to classification, one based on SVM and the other exploiting trained RBF NN. No obvious superiority between these two classifiers has been observed.

Acknowledgements. This study was partially supported by the CONACyT as a part of research project SEMAR No.11055.

\section{References}

1. Xiuping Jia, Richards, J.A., Gessner, W., Ricken, D.E.: Remote Sensing Digital Image Analysis. An Introduction. 3-rd edn. Springer-Verlag, Berlin Heidelberg New York (1999)

2. Kulemin, G.P., Zelensky, A.A., Astola, J.T., Lukin, V.V., Egiazarian, K.O., Kurekin, A.A., Ponomarenko, N.N., Abramov, S.K., Tsymbal, O.V., Goroshko, Y.A., Tarnavsky, Y.V.: Methods and Algorithms for Pre-processing and Classification of Multichannel Radar Remote Sensing Images, TICSP Series \#28, Tampere, Finland, TTY Monistamo (2004)

3. Aiazzi, B., Alparone, L., Baronti, S.: Near-Lossless Comprerssion of 3-D Optical Data. IEEE Trans. On Geoscience and Remote Sensing, V. 39, 11 (2001) 2547-2557

4. Kaarna, A., Zemcik, A., Kalviainen, H., Parkkinen, J.: Compression of multispectral remote sensing images using clustering and spectral reduction. IEEE Transactions on Geoscience and Remote Sensing, V. GRS-38, 2 (2000) 1073-1082

5. Du, Q., Chang, C.-I.: Linear Mixture Analysis-Based Compression for Hyperspectral Image Analysis. IEEE Transactions on Geoscience and Remote Sensing, V. GRS-42, 4, (2004) 875-891

6. Faulconbridge, R.I., Pickering, M.R., Ryan, M.R., Jia, X.: A New Approach to Controlling Compression-Induced Distortion of Hyperspectral Images. Proceedings of IGARSS, (2003) 1830-1832

7. Pal, M.D., Brislawn, C.M., Brumby, S.P.: Feature extraction from Hyperspectral Images Compressed Using the JPEG-2000 Standard. CD-ROM Proceedings of the Fifth IEEE Southwest Symp. On Image Analysis and Interpretation, (2002) 5 p. 
8. Kurekin, A., Dolia, A., Marshall, D., Lukin, V., Lever, K.: Mitigation of image impairments for multichannel remote sensing data fusion. Visual Information Processing XIV; Proc. of SPIE, V. 5817 (2005) 145-156

9. Ponomarenko, N., Lukin, V., Zriakhov, V., Egiazarian, K., Astola, J.: Lossy compression of images with additive noise. Proceedings of International Conference on Advanced Concepts for Intelligent Vision Systems, Antwerpen, Belgium (2005) 381-386

10. O.K. Al-Shaykh, R.M. Merserau: Lossy Compression of Noisy Images. IEEE Trans. On Image Processing, V. 712 (1998) 1641-1652

11. Salomon, D.: Data Compression. The Complete Reference. 3-rd edn. Springer-Verlag, Berlin Heidelberg New York (2004)

12. Taubman, D., Marcellin, M.: JPEG 2000: Image Compression Fundamentals. Standards and Practice. Kluwer, Boston (2002)

13. N.N. Ponomarenko, V.V. Lukin, K.O. Egiazarian, J.T. Astola: DCT Based High Quality Image Compression. Lecture notes in computer science, Vol. 3540, Springer-Verlag, Berlin Heidelberg New York (2005) 1177-1185

14. Ponomarenko N., Lukin V., Zriakhov M., Pogrebnyak O.: Methods for Lossy Compression of Images Corrupted by Multiplicative Noise. Proceedings of the International Conference "Modern Problems of Radioengineering, Telecommunications and Computer Science" (TCSET), (2006) 278-281

15. Carton-Vandecandelaere, M.-P., Vozel, B., Klaine, L., Chehdi, K.: Application to Multispectral Images of a Blind Identification System for Blur, Additive, Multiplicative and Impulse Noises. Proceedings of EUSIPCO, V. III (2002) 283-286

16. Lukin, V.V., Abramov, S.K., Vozel, B., Chehdi, K.: A method for blind automatic evaluation of noise variance in images based on bootstrap and myriad operations. Proceedings of SPIE/EUROPTO Symp. On Satellite Remote Sensing, SPIE V. 5982 (2005) 299-310

17. Ponomarenko, N., Lukin, V., Zriakhov, M., Egiazarian, K., Astola, J.: Estimation of accesible quality in noisy image compression. accepted to EUSIPCO, (2006) 4 p.

18. Christianini, N., Shave-Tailor, J.: An Introduction to Support Vector Machines and Other Kernel-based Learning Methods. Cambridge University Press (2000)

19. Duda, R.O., Harrt, P.E., Stork, D.G.: Pattern Classification. 2nd Edition. John Wiley and Sons (2001)

20. Schölkopf, B., Burges, J.C., Smola, A.J.: Advances in Kernel Methods: Support Vector Learning. MIT Press, Cambridge, MA (1999)

21. Hsu, C.-W., Lin, C.-J.: A comparison of methods for multi-class support vector machines. IEEE Transactions on Neural Networks, 13(2) (2002) 415-425

22. Bose, N.K., Liang, P.: Neural Network Fundamentals with Graphs, Algorithms and Applications. McGraw-Hill (1996)

23. Roli, F., Serpico, S.B., Bruzzone, L., Vernazza, G.: Classification of multispectral remotesensing images by neural networks. Proc. of the VIII European Signal Processing Conference, V. I (1996) 172-175

24. Foody, G.M.: Supervised image classification by MLP and RBF neural networks with and without an exhaustively defined set of classes. International Journal of Remote Sensing, 25, (15) (2004) 3091-1304

25. Zelenskyj, O., Kurekin, A., Lukin, V.: Techniques of Multichannel Radar Images Processing and Interpretation by Multilayer Neural Network. Proceedings of the Third AllUkrainian International Conference on Signal/ Image Processing and Pattern Recognition, (1996) 231-233 